\title{
Genetic Contribution to Retinopathy
}

Dear Sir,

Two recent publications in DIABETOLOGIA have reported on studies searching for relationships between the HLA and diabetic retinopathy. Deckert et al. [1] did not find any associations in a study of 36 Danish patients including 15 with proliferative retinopathy. Standl et al. [2], when studying 62 patients with insulin dependent diabetes (IDD) in Southern Germany, found the HLA antigen B8 associated with grade II retinopathy (Ballantyne's classification) but not with proliferative retinopathy. B18 was less common in those patients with an excess of B8. However, the duration of diabetes, especially for the "benign" (14 years) and grade II (16 years) groups was not long enough to rule out eventual development of proliferative changes. Furthermore, the $\mathrm{p}$ values reported should probably be corrected by multiplying by 3 (number of comparisons). This would make the difference for B8 not significant.

The findings of Standl et al. are in conflict with those obtained by us [3], when studying a larger sample of over 250 patients. In our study both the patients with proliferative retinopathy and those without had a duration of disease of over 20 years. We have found that $\mathrm{B} 7$ is significantly less common, and B15 is significantly more common in patients with proliferative retinopathy as compared to those without proliferative retinopathy. A similar association is suggested in the data of Deckert et al., where $10 / 15(67 \%)$ patients with proliferative retinopathy are B15. Unlike Standl et al. we did not find associations for $\mathrm{B} 8$ or $\mathrm{B} 18$.

Therefore, we agree that a genetic contribution to diabetic retinopathy is likely and may be reflected in
HLA associations. Since these associations often differ with the ethnic background of the population studied, it is possible that the associations between the HLA and retinopathy in Southern Germany will turn out to be different from those seen in our population. We also agree with Standl et al. that these findings should not be construed as minimizing the importance of metabolic control as a cause of diabetic microangiopathy. On the contrary, the use of both approaches simultaneously, i. e. more intensive treatment of those with predisposing genetic markers, may lead to the best results in preventing the complications of diabetes.

\section{References}

1. Deckert T, Egeberg J, Frimodt-Møller C, Sander E, Svejgaard A (1979) Basement membrane thickness, insulin antibodies and HLA antigens in long standing insulin dependent diabetics with and without severe retinopathy. Diabetologia 17: 91-96

2. Standl E, Dexel T, Lander T, Albert E, Scholz S (1980) HLA antigens and diabetic retinopathy: A different view warranted. Diabetologia 18: 79-80

3. Barbosa J, Ramsay R, Knobloch W, Cantrill H, Noreen H, King $R$, Yunis $E$ (in press) Genetic contribution to diabetic microangiopathy. The HLA and retinopathy. Am J Ophthalmol

Jose Barbosa

Division of Endocrinology and Metabolism

Department of Medicine

Robert Ramsay

Department of Ophthalmology

University of Minnesota

Minneapolis, MN 55455

USA 